\title{
Presenting features of COVID-19 in older people: relationships with frailty, inflammation and mortality
}

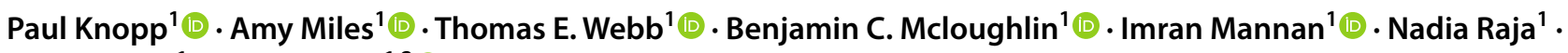 \\ Bettina Wan ${ }^{1} \cdot$ Daniel Davis ${ }^{1,2}$
}

Received: 29 May 2020 / Accepted: 20 July 2020 / Published online: 30 July 2020

(c) The Author(s) 2020

\section{Key summary points}

Aim To characterise symptoms, key findings and clinical outcomes in older adults with COVID-19.

Findings $12 \%$ of older individuals did not present with classical COVID-19 symptoms, though fever, dyspnoea, delirium and raised inflammation were associated with higher mortality. Compared with fitter older individuals, some measures of immune activity were lower in frailer patients.

Message COVID-19 may present without cardinal symptoms as well as implicate a possible role for age-related changes in immunity in mediating the relationship between frailty and mortality.

\begin{abstract}
Purpose To describe the clinical features of COVID-19 in older adults, and relate these to outcomes.

Methods A cohort study of 217 individuals (median age 80, IQR 74-85 years; 62\% men) hospitalised with COVID-19, followed up for all-cause mortality, was conducted. Secondary outcomes included cognitive and physical function at discharge. C-reactive protein and neutrophil:lymphocyte ratio were used as measures of immune activity.

Results Cardinal COVID-19 symptoms (fever, dyspnoea, cough) were common but not universal. Inflammation on hospitalisation was lower in frail older adults. Fever, dyspnoea, delirium and inflammation were associated with mortality. Delirium at presentation was an independent risk factor for cognitive decline at discharge.

Conclusions COVID-19 may present without cardinal symptoms as well as implicate a possible role for age-related changes in immunity in mediating the relationship between frailty and mortality.
\end{abstract}

Keywords COVID-19 $\cdot$ Immune dysfunction $\cdot$ Epidemiology $\cdot$ Mortality $\cdot$ Post-hospitalisation outcomes

\section{Introduction}

As healthcare systems around the world start to respond to SARS-CoV-2, a major consideration is the apparent agerelated heterogeneity in presentation, treatment responsiveness and clinical outcomes [1]. COVID-19 in older people, prima facie, may present in the absence of classical

Daniel Davis

daniel.davis@ucl.ac.uk

1 Department of Medicine for the Elderly, University College London Hospitals NHS Foundation Trust, 1-19 Torrington Place, London WC1E 7HB, UK

2 Department of Population Science and Experimental Medicine, MRC Unit for Lifelong Health and Ageing at UCL, London, UK symptoms, progress more rapidly to severe disease, have poorer intensive care outcomes, longer inpatient stay and higher mortality $[2,3]$. Nonetheless, questions remain as to which presenting features have greatest impact on these outcomes in older people. Recognising this may lead to better clinical care, as well as forming the basis for new services for older people after COVID-19.

Given this urgent need to understand COVID-19 in older people, we set out to describe the clinical, laboratory and radiological features in a series of older individuals hospitalised with COVID-19 in a large urban hospital. 


\section{Methods}

\section{Study design and participants}

As previously described, we undertook a prospective cohort study of patients aged $\geq 70$ years admitted to University College Hospital diagnosed with COVID-19 up until 23rd April 2020 [4]. Patients were included if they tested positive for SARS-CoV-2 on reverse-transcriptase polymerase chain reaction from a combined oropharyngeal and nasal swab. We also included swab-negative participants with a clinical diagnosis of COVID-19 on review of clinical, laboratory and radiological findings by a specialist infectious diseases team.

\section{Outcome}

Primary outcome was all-cause mortality recorded during admission or if occurring after discharge updated from NHS Spine, a collection of local and national demographic databases. Vital status was followed up until 13th May 2020. Secondary outcomes included any decreased cognitive or physical function at discharge.

\section{Clinical measures}

We recorded demographic data on age, sex and ethnicity. Presenting features included fever, cough, dyspnoea, and gastrointestinal symptoms, along with any geriatric syndromes: delirium, reduced mobility, and falls. The Clinical Frailty Scale (CFS) was used to quantify frailty, with scores assigned by specialist geriatricians. We measured C-reactive protein (CRP) and neutrophil:lymphocyte ratios as indicators of immune activity and noted the presence of any radiological abnormalities reported by specialist radiologists.

\section{Ethics approvals}

These analyses were conducted as part of a service evaluation project and individual consent was not necessary as determined by the NHS Health Research Authority (HRA), the regulatory body for medical research for England, UK. The HRA has the Research Ethics Service as one of its core functions and they determined the project was exempt from the need to obtain approval from an NHS Research Ethics Committee [5].

\section{Statistical analyses}

We regarded the presenting features as being present or absent. We examined distributions of CRP and neutrophil:lymphocyte ratios at graded levels of frailty (CFS
1-3; CFS 4-6; CFS 7-9), with differences in median values assessed using the Kruskal-Wallis test. We estimated associations between presenting clinical, laboratory or radiological features with mortality in a series of univariable and multivariable Cox proportional hazards models. To estimate associations with increased rehabilitation needs (cognitive and/or physical), we used logistic regression. Post-estimation procedures included Schoenfeld residuals and Hosmer-Lemeshow tests for heteroskedasticity. Stata 14.1 (StataCorp, Texas, USA) was used for all analyses.

\section{Results}

We identified 217 individuals aged $\geq 70$ years hospitalised with COVID-19. Median age of patients was 80 years (range 70-99 years), $62 \%$ were male and a range of pre-morbid frailty was identified (Table 1). The majority of patients had no formal package of community care $(n=154,71 \%)$ and eight patients $(4 \%)$ were admitted from residential care homes. 72 individuals (33\%) were living with definite or probable dementia (Table 1).

Table 1 Characteristics and presenting symptoms of older adults ( $\geq 70$ years) with COVID-19 admitted to hospital

\begin{tabular}{lc}
\hline Clinical features & $\begin{array}{c}\text { COVID- } \\
19 \text { cohort } \\
(n=217)\end{array}$ \\
\hline Age (mean, SD) & $80(6.8)$ \\
Male & $134(62 \%)$ \\
Clinical Frailty Scale tertiles & \\
$1-3$ & $68(31 \%)$ \\
$4-6$ & $98(45 \%)$ \\
$7-9$ & $49(23 \%)$ \\
Package of care & \\
None & $154(71 \%)$ \\
Weekly & $1(0.5 \%)$ \\
Once daily & $6(2.8 \%)$ \\
Twice daily & $14(6.5 \%)$ \\
Three times daily & $9(4.2 \%)$ \\
Four times daily & $17(7.8 \%)$ \\
24 h care & $16(7.4 \%)$ \\
Care home resident & $8(4 \%)$ \\
Dementia & $72(33 \%)$ \\
Presenting symptoms & \\
Fever & $157(72 \%)$ \\
Dyspnoea & $143(66 \%)$ \\
Cough & $130(60 \%)$ \\
Gastrointestinal disturbance & $7(3 \%)$ \\
Fall & $60(28 \%)$ \\
Reduced mobility & $97(45 \%)$ \\
Delirium & $64(29 \%)$ \\
\hline &
\end{tabular}


On admission, symptoms of fever, dyspnoea and cough were common $(n=157,72 \% ; \mathrm{n}=143,66 \% ; n=130,60 \%$ respectively) (Table 1). Symptoms of gastrointestinal disturbance were less frequent $(n=7,3 \%)$. Some individuals were admitted without any of these cardinal COVID-19 symptoms $(n=25,12 \%)$, instead presenting with one or more frailty syndrome (reduced mobility, falls or delirium). Figure 1 details the combinations of presenting symptoms observed in our sample, with cardinal and frailty symptoms given separately.
The CRP range was $0-480 \mathrm{mg} / \mathrm{L}$ (median 92, IQR $30-172 \mathrm{mg} / \mathrm{L})$. Neutrophils and lymphocytes ranged from $0.05-36 \times 10^{9} / \mathrm{L}$ (median 6.1 , IQR $4.2-8.6 \times 10^{9} / \mathrm{L}$ ) ti $0.07-8.0 \times 10^{9} / \mathrm{L}$ (median 0.9 , IQR $0.6-1.5 \times 10^{9} / \mathrm{L}$ ), respectively. With higher CFS scores, there were decreases in CRP $(p<0.01)$ and neutrophil:lymphocyte ratio $(p=0.05)$ (Table 2 and Fig. 2).

The contribution of demographic data, clinical presentation and inflammatory markers on admission to mortality was assessed in univariable and multivariable
Fig. 1 Euler diagram of common presenting clinical symptoms (a) and frailty syndromes (b) in hospitalised older adults with COVID-19. The denominator for each figure separately is the total sample $(n=217)$
A

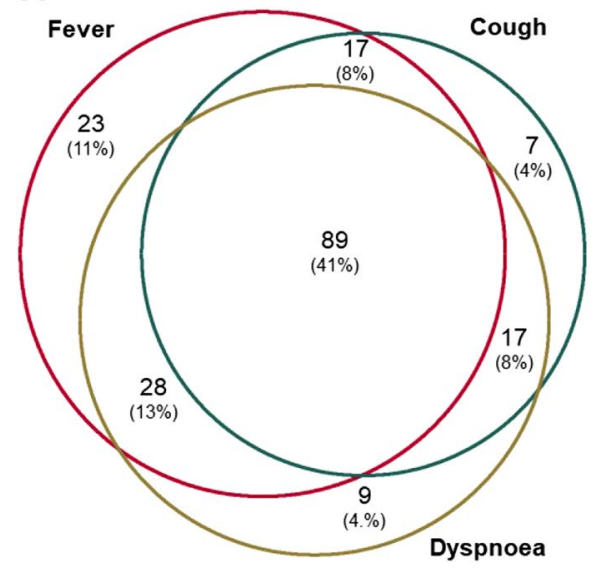

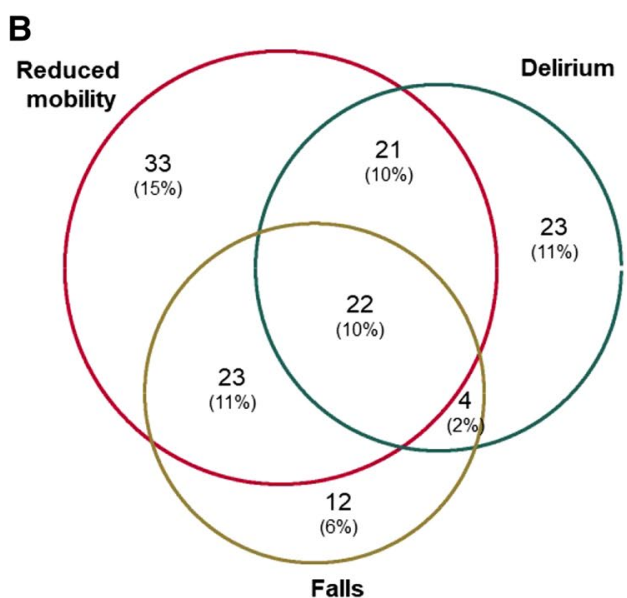

Table 2 Distribution of C-reactive protein (CRP) and neutrophil:lymphocyte ratio in mild (Clinical Frailty Scale 1-3), moderate (Clinical Frailty Scale 4-6) and severely frail (Clinical Frailty Scale 7-9) older COVID-19 patients

\begin{tabular}{|c|c|c|c|c|}
\hline & CFS $1-3$ & CFS 4-6 & CFS 7-9 & $p$ \\
\hline CRP, mg/L (median, IQR) & $120(65-250)$ & $69(23-169)$ & $65(17-119)$ & $<0.01$ \\
\hline Neutrophil:lymphocyte (median, IQR) & $7.2(4.6-17.1)$ & $6.3(3.4-9.9)$ & $5.9(2.9-9.2)$ & 0.05 \\
\hline
\end{tabular}

Fig. 2 Relationship between immune activity on hospitalisation by degree of frailty and possible divergent routes to mortality




models (Table 3). There was an age-related increase in mortality (HR 1.1, 95\% CI 1.0-1.1, p <0.01), but neither sex nor frailty was associated with mortality (Table 3 ). Of classical COVID-19 symptoms, fever (HR 1.97, 95\% CI $1.4-3.4, p=0.02$ ) or dyspnoea (multivariable HR $2.0,95 \%$ CI $1.2-3.3, p=0.01$ ) at presentation was associated with increased mortality. For frailty syndromes, delirium was associated with mortality (HR 1.9, 95\% CI 1.2-3.0, $p<0.01$ ) (Table 3). With rising CRP or higher neutrophil:lymphocyte ratio, there was a corresponding increase in likelihood of death (Table 3).

High dependency unit level care and progression to non-invasive ventilation (NIV) or intubation demonstrated higher age-sex-frailty adjusted mortality (HR 3.5, 95\% CI, 2.3-5.6, $p<0.01)$.

In terms of increased rehabilitation needs (evidence of cognitive or physical decline at discharge), only delirium was associated with new or worsening of cognitive impairment in models adjusted for age, sex, dementia and premorbid frailty (OR 44, 95\% CI 7.4-260). No other admission parameters were associated with cognitive decline or physical decline at discharge, including premorbid dementia.

\section{Discussion}

We described the clinical characteristics and outcomes of older adults admitted to a large London hospital in the first 100 days of the UK COVID-19 outbreak. Frailty syndromes were common presentations, sometimes in the absence of classical COVID-19 symptoms. Some presenting features, fever, dyspnoea, or delirium, but not frailty, were associated with increased mortality. However, frailty was associated with a lower degree of inflammation on admission. Taken together, these results quantify the degree to which COVID19 may present without cardinal symptoms as well as implicate a possible role for age-related changes in immunity (immunosenescence) in mediating the relationship between frailty and mortality.

Our findings should be treated with caution. Our data come from a single site in an urban population in the context of the UK National Health Service (NHS) undergoing restructuring to prepare for the COVID-19 pandemic, limiting generalisation to different healthcare systems. In particular, the need for 'further rehabilitation on discharge' will be specific to the local interface between secondary and community care. Furthermore, as a hospitalised cohort, clinical features and their relation to outcome might vary in population samples. There was only one individual with CFS score of 9 included in the group 'CFS 7-9', though the continuum
Table 3 Univariable and multivariable analyses estimating the association of presenting features on mortality in older patients with COVID19

\begin{tabular}{|c|c|c|c|c|c|c|}
\hline & \multicolumn{3}{|c|}{ Univariable models } & \multicolumn{3}{|c|}{ Multivariable model } \\
\hline & HR & $95 \% \mathrm{CI}$ & $p$ & HR & $95 \% \mathrm{CI}$ & $p$ \\
\hline Age & 1.03 & $(1.01-1.06)$ & 0.02 & 1.06 & $(1.03-1.09)$ & $<0.01$ \\
\hline Sex & 1.25 & $(0.84-1.86)$ & 0.26 & 1.22 & $(0.80-1.87)$ & 0.36 \\
\hline CFS & 1.02 & $(0.93-1.12)$ & 0.71 & & & \\
\hline Cough & 1.45 & $(0.98-2.15)$ & 0.07 & 1.17 & $(0.75-1.84)$ & 0.49 \\
\hline Fever & 1.75 & $(1.09-2.82)$ & 0.02 & 1.97 & $(1.14-3.41)$ & 0.02 \\
\hline Dyspnoea & 2.14 & $(1.37-3.35)$ & $<0.01$ & 1.96 & $(1.16-3.29)$ & 0.01 \\
\hline Gastrointestinal & 0.43 & $(0.11-1.74)$ & 0.24 & 0.45 & $(0.11-1.91)$ & 0.28 \\
\hline Imaging abnormalities & 1.55 & $(1.08-2.22)$ & 0.02 & 1.23 & $(0.81-1.86)$ & 0.33 \\
\hline Falls & 0.91 & $(0.59-1.4)$ & 0.68 & 0.89 & $(0.55-1.44)$ & 0.64 \\
\hline Reduced mobility & 1.1 & $(0.76-1.6)$ & 0.61 & 0.75 & $(0.47-1.2)$ & 0.23 \\
\hline Delirium & 1.28 & $(0.87-1.88)$ & 0.21 & 1.91 & $(1.2-3.04)$ & $<0.01$ \\
\hline CRP (quartiles) & & & $<0.01$ & & & $<0.01$ \\
\hline 2 & 1.3 & $(0.73-2.31)$ & 0.38 & 1.56 & $(0.81-3)$ & 0.18 \\
\hline 3 & 1.88 & $(1.07-3.3)$ & 0.03 & 2.52 & $(1.29-4.94)$ & $<0.01$ \\
\hline 4 & 2.37 & $(1.38-4.06)$ & $<0.01$ & 3.03 & $(1.6-5.74)$ & 0.01 \\
\hline $\begin{array}{l}\text { Neurotophil: lymphocytes } \\
\text { (quartiles) }\end{array}$ & & & 0.13 & & & 0.04 \\
\hline 2 & 0.98 & $(0.58-1.68)$ & 0.96 & 0.86 & $(0.47-1.59)$ & 0.64 \\
\hline 3 & 0.82 & $(0.48-1.42)$ & 0.49 & 0.54 & $(0.29-1)$ & 0.05 \\
\hline 4 & 1.52 & $(0.92-2.51)$ & 0.1 & 1.23 & $(0.67-2.25)$ & 0.5 \\
\hline
\end{tabular}

$H R$ hazard ratio; $C I$ confidence interval; $C F S$ Clinical Frailty Scale; $C R P$ C-reactive protein 
of frailty is more properly considered as ranging from 1 to 8 . Our measures of immune activity were derived from routinely available laboratory tests on presentation to acute care, and can only tangentially taken to be markers of immunosenescence. Nonetheless, we had the advantage of specialist geriatrician review of all electronic patient records, which allowed us to ascertain outcomes in near real time.

The prevalence of cardinal symptoms appear to be similar in older and younger patients, though delirium is more common here than in ISARIC where around $25 \%$ presented with 'confusion'. Our data indicate that fever and dyspnoea may be important prognostic signs. The clinical significance of delirium has been observed in other COVID-19 cohorts, including from our own hospital [6, 7]. However, the inverse relationship between CRP on admission and frailty has not previously been noted in COVID-19.

These findings add to the growing descriptive data characterising COVID-19 presentations in older adults $[8,9]$ and may help inform prognosis at the point of hospital admission. Overt inflammatory activation has been implicated in COVID-19 pathogenesis [10-13], but it is not clear the extent to which this might operate in older adults living with frailty. Frailty and chronic inflammation are linked to immunosenescence and may influence response to infection and subsequent immunity [14-16]. Our finding of lower levels of inflammation in frail patients indirectly supports the possibility that background frailty and immunosenescence could constrain the acute inflammation evident in COVID-19 (Fig. 2). Whether this accounts for the apparent excess mortality in fitter patients remains speculative [4], though, if borne out by further research, has implications for future therapeutic and vaccine strategies.

COVID-19 disproportionately affects older people, warranting a co-ordinated global response. Even in this early stage of understanding the disease, biological complexities that come with ageing are particularly apparent in this population. This then becomes an opportunity, indeed a responsibility, for professionals with expertise in clinical and research practice in older people to intensify their efforts on COVID-19.

Author contributions PK, AM, TW, NR, IM, and BM collected the primary data. DD undertook the statistical analyses and both BW and DD had oversight of the project. PK drafted the first version of the manuscript. All authors contributed to revision and intellectual content of the final submission.

Funding Daniel Davis is funded through a Wellcome Intermediate Clinical Fellowship (WT107467).

Data availability On request.

Code availability On request.

\section{Compliance with ethical standards}

Conflict of interest The authors declare that they have no conflict of interest.

Ethical approval The HRA has the Research Ethics Service as one of its core functions and they determined the project was exempt from the need to obtain approval from an NHS Research Ethics Committee. https://www.hra.nhs.uk/about-us/committees-and-services/res-andrecs/.

Informed consent These analyses were conducted as part of a service evaluation project and individual consent was not necessary as determined by the NHS Health Research Authority (HRA), the regulatory body for medical research for England, UK.

Open Access This article is licensed under a Creative Commons Attribution 4.0 International License, which permits use, sharing, adaptation, distribution and reproduction in any medium or format, as long as you give appropriate credit to the original author(s) and the source, provide a link to the Creative Commons licence, and indicate if changes were made. The images or other third party material in this article are included in the article's Creative Commons licence, unless indicated otherwise in a credit line to the material. If material is not included in the article's Creative Commons licence and your intended use is not permitted by statutory regulation or exceeds the permitted use, you will need to obtain permission directly from the copyright holder. To view a copy of this licence, visit http://creativecommons.org/licenses/by/4.0/.

\section{References}

1. Lloyd-Sherlock P, Ebrahim S, Geffen L, McKee M (2020) Bearing the brunt of covid-19: older people in low and middle income countries. BMJ 368:m1052. https://doi.org/10.1136/bmj.m1052

2. Wu Z, McGoogan JM (2019) Characteristics of and important lessons from the Coronavirus Disease 2019 (COVID-19) outbreak in China: summary of a report of 72314 cases from the Chinese Center for Disease Control and Prevention. JAMA. https://doi. org/10.1001/jama.2020.2648Accessed 25 July 2020

3. Sun P, Qie S, Liu Z, Ren J, Li K, Xi J (2020) Clinical characteristics of hospitalized patients with SARS-CoV-2 infection: a single arm meta-analysis. J Med Virol. https://doi.org/10.1002/ jmv. 25735

4. Miles A, Webb T, Mcloughlin B, Mannan I, Rather A, Knopp P, Davis D (2020) Outcomes from COVID-19 across the range of frailty: excess mortality in fitter older people. Eur Geriatr Med. https://doi.org/10.1007/s41999-020-00354-7

5. www.hra.nhs.uk/about-us/committees-and-services/res-and-recs. Accessed 25 July 2020

6. Docherty AB, Harrison EM, Green CA, Hardwick HE, Pius R, Norman L et al (2020) Features of 20133 UK patients in hospital with covid-19 using the ISARIC WHO Clinical Characterisation Protocol: prospective observational cohort study. BMJ 369:m1985. https://doi.org/10.1136/bmj.m1985

7. Mcloughlin BC, Miles A, Webb T, Knopp P, Eyres C, Fabbri A et al (2020) Functional and cognitive outcomes after COVID-19 delirium. Eur Geriatr Med. https://doi.org/10.1007/s41999-02000353-8

8. Liu K, Chen Y, Lin R, Han K (2020) Clinical features of COVID19 in elderly patients: a comparison with young and middle-aged 
patients. J Infect 80(6):e14-e18. https://doi.org/10.1016/j. jinf.2020.03.005

9. Wang L, He W, Yu X, Hu D, Bao M, Liu H et al (2020) Coronavirus disease 2019 in elderly patients: characteristics and prognostic factors based on 4-week follow-up. J Infect 80(6):639-645. https ://doi.org/10.1016/j.jinf.2020.03.019

10. Mehta P, McAuley DF, Brown M, Sanchez E, Tattersall RS, Manson JJ et al (2020) COVID-19: consider cytokine storm syndromes and immunosuppression. Lancet 395(10229):1033-1034. https:// doi.org/10.1016/S0140-6736(20)30628-0

11. Herold T, Jurinovic V, Arnreich C, Hellmuth JC, von BergweltBaildon M, Klein M et al (2020) Level of IL-6 predicts respiratory failure in hospitalized symptomatic COVID-19 patients. J Allergy Clin Immunol 146:128-136

12. Moore JB, June CH (2020) Cytokine release syndrome in severe COVID-19. Science 368(6490):473-474. https://doi.org/10.1126/ science.abb8925

13. Tay MZ, Poh CM, Renia L, MacAry PA, Ng LFP (2020) The trinity of COVID-19: immunity, inflammation and intervention. Nat Rev Immunol. https://doi.org/10.1038/s41577-020-0311-8
14. Wilson D, Jackson T, Sapey E, Lord JM (2017) Frailty and sarcopenia: The potential role of an aged immune system. Ageing Res Rev 36:1-10. https://doi.org/10.1016/j.arr.2017.01.006

15. Bonafe M, Prattichizzo F, Giuliani A, Storci G, Sabbatinelli J, Olivieri F (2020) Inflamm-aging: why older men are the most susceptible to SARS-CoV-2 complicated outcomes. Cytokine Growth Factor Rev. https://doi.org/10.1016/j.cytogfr.2020.04.005

16. Oh SJ, Lee JK, Shin OS (2019) Aging and the immune system: the impact of immunosenescence on viral infection, immunity and vaccine immunogenicity. Immune Netw 19(6):e37. https:// doi.org/10.4110/in.2019.19.e37

Publisher's Note Springer Nature remains neutral with regard to jurisdictional claims in published maps and institutional affiliations. 